\title{
Estimação de Parâmetros Genéticos em Bovinos de Corte Utilizando os Métodos de Máxima Verossimilhança Restrita e $\Re^{1}$
}

\section{Marcelo Hessel Van Melis ${ }^{2}$, Joanir Pereira Eler ${ }^{3}$, Josineudson Augusto II de Vasconcelos Silva ${ }^{4}$, José Bento Sterman Ferraz ${ }^{3}$}

\footnotetext{
RESUMO - Com o objetivo de comparar as estimativas de parâmetros genéticos obtidas pelos métodos de máxima verossimilhança restrita (REML) e $\mathfrak{R}$, foram realizadas análises uni-características sob modelo animal, para os quais foi utilizado um conjunto de dados da raça Nelore. Os modelos estatísticos incluíram os efeitos fixos de grupo de contemporâneos (fazenda, ano de nascimento, sexo e grupos de manejo à desmama e ao sobreano) e idade do animal (covariável linear), e como aleatórios foram considerados os efeitos genético aditivo direto e residual. As estimativas dos coeficientes de herdabilidade foram 0,36 e 0,39 para peso aos 18 meses (PESSOB); 0,27 e 0,29 para ganho de peso da desmama aos 18 meses (GP345); 0,22 e 0,21 para conformação (CONF); 0,21 e 0,21 para precocidade de acabamento (PREC); e 0,22 e 0,22 para musculosidade (MUSC), respectivamente, pelo REML e método R. Em todas as estimativas de herdabilidade, o erro-padrão foi menor ou igual a 0,02 . As diferenças entre as estimativas de tendência genética obtidas pelos dois métodos foram pequenas e as correlações de Spearman entre os valores genéticos preditos usando os componentes de variância obtidos por ambos os métodos foram iguais a 1 para todas as características analisadas. Os resultados sugerem que os métodos REML e $\Re$ fornecem os mesmos resultados e que o método $\mathfrak{R}$ pode ser utilizado como uma opção ao REML em análises uni-características, principalmente pela menor demanda computacional.
}

Palavras-chave: características de crescimento, escore visual, Método $\Re$, Nelore, parâmetros genéticos, REML

\section{Estimate of Genetic Parameters in Beef Cattle Using Restricted Maximun Likelihood and Method $\Re$}

\begin{abstract}
The objective of this paper was to compare the estimates of genetic parameters obtained by REML and method $\Re$. Animal model single-trait analyses were performed using a data set of Nellore cattle. Statistical models included fixed effects of contemporary group (herd, year, sex and management groups at weaning and 550 days) and age of animal (covariable-linear effect), and additive genetic and residual random effects. Heritability estimates were 0.36 and 0.39 for weight at 550 days (W550); 0.27 and 0.29 for weight gain from weaning to 550 days (WG345); 0.22 and 0.21 for conformation (CONF); 0.21 and 0.21 for precocity (PREC) and 0.22 and 0.22 for muscling (MUSC), respectively, by REML and method $\Re$. For all heritability estimates, standard errors were lower than or equal to 0.02 . The differences in the estimates of genetic trend obtained from the two methods were low for all traits. Spearman rank correlation between breeding values obtained in the analyses using the variance components obtained by REML and method $\Re$ were equal to 1 for all traits. The results suggest that REML and method $\Re$ provide similar results and that method $\Re$ can be used as an alternative to REML in single-trait analyses, mainly due to lower computational requirements.
\end{abstract}

Key Words: genetic parameters, growth traits, Method $\Re$, Nellore, REML, visual score

\section{Introdução}

Os componentes de variância, importantes na predição do mérito genético dos indivíduos, têm sido estimados por diferentes métodos que evoluem à medida que novas teorias e novas técnicas computacionais são desenvolvidas.

Segundo Nelsen et al. (1986), o método de estimação pode ser um fator importante na obtenção dos parâmetros genéticos, juntamente com o estabeleci- mento de um modelo matemático que descreva corretamente os dados e com a inclusão de fatores importantes, como a raça (De Rose et al., 1988), o sexo (Schaeffer \& Wilton, 1981), o manejo (Tess et al., 1984) e a origem dos dados (Wright et al., 1987).

O método de máxima verossimilhança restrita (REML) tem sido o preferido para a estimação de componentes de variância e covariância em melhoramento genético animal pelas suas propriedades teóricas desejáveis, enquanto o método $\mathfrak{R}$ surgiu

\footnotetext{
${ }^{1}$ Pesquisa financiada pela FAPESP e pelo CNPq.

${ }^{2}$ MS em Zootecnia, FZEA/USP, Pirassununga, SP, CP. 23, CEP: 13635-970, E.mail: mhvmelis@yahoo.com

3 Professores da FZEA/USP. E.mail: joapeler@usp.br; jbferraz@usp.br

${ }^{4}$ Pós-doutorando, FZEA/USP. E.mail: jaugustovs@yahoo.com
} 
como alternativa de menor custo computacional.

O desenvolvimento teórico do método $\Re$ iniciou-se em um estudo para detecção de viés em predições genéticas (Reverter et al., 1994a). Investigando a ocorrência de mudanças nas predições genéticas conforme os valores de acurácia aumentavam ao longo do tempo, os autores demonstraram que o valor esperado da regressão das predições genéticas recente (mais acurada) sobre a prévia (menos acurada) se iguala a um. Qualquer desvio observado em relação à unidade indica a existência de viés. Esta mesma pesquisa concluiu que, ao passo que a quantidade de observações obtidas entre análises aumenta, a covariância entre as predições provenientes de cada análise permanece constante e igual a variância da predição menos acurada.

O método $\Re$ foi desenvolvido por Reverter et al. (1994b) e tem como principal vantagem a utilização de um algoritmo que requer apenas os valores genéticos preditos, os quais podem ser obtidos por método iterativo, não exigindo a decomposição de Cholesky do sistema de equações (DF-REML), sua inversa (EM-REML) ou derivadas (Newton-RaphsonREML). Isto implica em maior velocidade no processamento e menor demanda de memória nos computadores. Esta praticidade tem feito do método $\mathfrak{R}$ o método de escolha para estimação de componentes de variância de dominância em modelos complexos e grandes (Misztal, 1997; Misztal et al., 1997; Culbertson et al., 1998).

Segundo Reverter et al. (1994b), o método $\Re$ tem propriedades desejáveis de convergência, precisão e praticidade computacional. Misztal (1997) ressaltou que entre outras propriedades desejáveis, o método $\mathfrak{R}$ é robusto ao viés causado pelos acasalamentos não-aleatórios, por considerar a matriz de parentesco, e que é tão robusto quanto o REML à seleção causada pelas substituições não aleatórias e pelo controle seletivo de dados.

Embora as propriedades teóricas do método $\mathfrak{R}$ não sejam perfeitamente conhecidas (Schaeffer, 1998; Druet et al., 2001), Reverter et al. (1994b) relataram que as estimativas obtidas pelo método $\mathfrak{R}$ devem ser congruentes com aquelas obtidas pelo REML em conseqüência da similaridade das formas quadráticas usadas por ambos.

Cantet \& Birchmeier (1998) e Schenckel \& Schaeffer (2000), comparando métodos de estimação de parâmetros genéticos em bancos de dados simulados, relataram que as estimativas obtidas pelo método $\Re$ foram superestimadas e, portanto, viesadas, com seleção genética baseada na metodologia BLUP. Por outro lado, Duangjinda et al. (2001) relataram que as estimativas pelo método $\Re$ foram não viesadas em populações em que a seleção era baseada nos valores genéticos obtidos pela metodologia BLUP ou em que a seleção fenotípica era praticada dentro de grupos de contemporâneos. Kaiser \& Golden (1994), usando o método $\Re$ em populações selecionadas, também encontraram estimativas não viesadas.

O objetivo deste estudo foi comparar os métodos REML e $\mathfrak{R}$ pela estimação de coeficientes de herdabilidade, tendências genéticas e pela correlação de Spearman entre os valores genéticos obtidos para características produtivas e de escore visual, numa população grande da raça Nelore selecionada pela metodologia BLUP.

\section{Material e Métodos}

\section{Dados}

As informações utilizadas neste estudo foram provenientes de doze fazendas situadas nos Estados de São Paulo, Mato Grosso do Sul e Goiás, e pertencentes ao Programa de Melhoramento Genético da Agro-Pecuária CFM Ltda. Os animais foram criados em regime exclusivo de pastagem, recebendo suplementação mineral apenas no período mais crítico do ano.

Foram analisadas as características produtivas peso aos 18 meses (PESSOB) e ganho de peso da desmama aos 18 meses (GP345) e as de escore visual, conformação (CONF), precocidade (PREC) e musculosidade (MUSC) aos 18 meses.

O escore visual de conformação (CONF) considera o comprimento, a largura, a profundidade e a aparência geral do indivíduo. A precocidade (PREC) é uma medida da capacidade do indivíduo em armazenar reservas de gordura, indicando ser mais precoce em sua terminação. A musculosidade (MUSC) leva em conta a massa muscular presente no animal, sendo uma das avaliações visuais que vem merecendo preocupação de diversos criadores que visam selecionar animais adequados aos padrões pedidos pela indústria da carne bovina.

O conjunto de dados inicial consistiu de 308.352 registros de animais da raça Nelore nascidos entre 1984 e 1999. As pesagens foram antecedidas por jejum de 12 horas. Para estimação dos coeficientes de herdabilidade, foram considerados somente animais com pais conhecidos. Para as características 
produtivas, os grupos de contemporâneos (GC) com apenas uma observação foram eliminados, assim como os animais que estavam fora dos limites da média \pm três desvios-padrão dentro de cada GC. Para as características de escore visual foram eliminados os GC com menos de cinco observações e também os GC que não possuíam variabilidade, ou seja, aqueles em que todos os animais possuíam o mesmo escore visual. A definição dos GC incluiu fazenda, ano de nascimento, sexo e grupos de manejo à desmama e ao sobreano para todas as características avaliadas.

Os arquivos de dados editados são mostrados na Tabela 1.

Os valores das características não foram préajustados. $\mathrm{O}$ ajuste para idade foi feito pela inclusão da idade como covariável no modelo estatístico.

O GP345 refere-se ao ganho de peso total em um período de 345 dias da desmama aos 18 meses de idade e foi obtido pela seguinte equação:

$$
\text { GP345 }=\left(\frac{\text { PESSOB }- \text { PESDES }}{\text { IDASOB }- \text { IDADES }}\right) \times 345 \text {, em que: }
$$

em que: GP345 é o ganho de peso da desmama aos 18 meses; PESSOB, o peso aos 18 meses; PESDES, o peso à desmama; IDASOB, a idade da medida aos 18 meses; e IDADES, a idade da medida à desmama.

Para obtenção do GP345, foram considerados somente os animais que tiveram informações ao sobreano e à desmama, respeitando-se intervalos de idade de 140 a 270 dias, para pesagem à desmama e de 450 a 650 dias, para pesagem ao sobreano.

Os escores visuais de CONF, PREC e MUSC foram avaliados ao sobreano e corresponderam às atribuições de notas aos animais, para cada uma das características em questão, sendo que os escores variaram em uma escala de 1 (péssimo) a 9 (excelente). As notas foram atribuídas em observações dentro do grupo de contemporâneos. Cada característica foi avaliada individualmente.

\section{Modelo}

O desempenho de cada animal pode ser explicado pelo seguinte modelo matricial:

$$
\mathrm{y}=\mathrm{Xb}+\mathrm{Za}+\mathrm{e},
$$

em que: $\mathrm{y}=$ vetor das variáveis dependentes (PESSOB, GP345, CONF, PREC e MUSC); $\mathrm{b}=$ vetor dos efeitos fixos (GC e covariável); $\mathrm{X}=$ matriz de incidência que associa elementos de $\mathrm{b}$ a y; $\mathrm{a}=$ vetor dos efeitos aleatórios de valor genético aditivo direto do animal, $\mathrm{a} \sim \operatorname{MVN}\left(0, A \sigma^{2}\right) ; \mathrm{Z}=$ matriz de incidência que associa elementos de a a y; e e = vetor dos efeitos aleatórios residuais, e $\sim \operatorname{MVN}(0, I \sigma 2)$.

A idade à pesagem e/ou medição foi utilizada como covariável (efeito linear), sendo que o modelo para a característica GP345 incluiu as idades à desmama e ao sobreano. Para a análise genética, a metodologia utilizada foi a melhor predição linear não-viesada utilizando as equações de modelos mistos, com utilização do modelo animal.

Tabela 1 - Descrição dos arquivos de dados editados para bovinos da raça Nelore

\begin{tabular}{|c|c|c|c|c|c|}
\hline & \multicolumn{5}{|c|}{$\begin{array}{c}\text { Característica } \\
\text { Trait } \\
\end{array}$} \\
\hline & $\begin{array}{c}\text { PESSOB } \\
W 550 \\
(\mathrm{~kg}) \\
(\mathrm{kg}) \\
\end{array}$ & $\begin{array}{c}\text { GP345 } \\
W G 345 \\
(\mathrm{~kg}) \\
(\mathrm{kg}) \\
\end{array}$ & $\begin{array}{c}\text { CONF } \\
\text { CONF } \\
\text { (escore) } \\
\text { (score) } \\
\end{array}$ & $\begin{array}{c}\text { PREC } \\
\text { PREC } \\
\text { (escore) } \\
\text { (score) } \\
\end{array}$ & $\begin{array}{c}\text { MUSC } \\
\text { MUSC } \\
\text { (escore) } \\
\text { (score) } \\
\end{array}$ \\
\hline$\overline{\mathrm{NA}(N A)}$ & 44.075 & 43.759 & 34.040 & 34.055 & 34.093 \\
\hline $\mathrm{NGC}(N C G)$ & 1.476 & 1.470 & 918 & 919 & 923 \\
\hline NAMP (NARM) & 74.788 & 74.256 & 61.412 & 61.350 & 61.417 \\
\hline Média (Mean) & 309,88 & 117,38 & 5,97 & 6,02 & 5,90 \\
\hline $\mathrm{DP}(S D)$ & 47,43 & 33,34 & 1,02 & 1,04 & 1,03 \\
\hline $\mathrm{CV}(C V)$ & 15,31 & 28,40 & 17,03 & 17,34 & 17,39 \\
\hline $\mathrm{MIN}(M I N)$ & 100,00 & 1,96 & 2,00 & 2,00 & 2,00 \\
\hline $\operatorname{MAX}(M A X)$ & 614,00 & 334,34 & 9,00 & 9,00 & 9,00 \\
\hline
\end{tabular}

Table 1 - Summary of edited data sets for Nelore beef cattle

PESSOB - peso ao sobreano; GP345 - ganho de peso da desmama ao sobreano; CONF - conformação; PREC - precocidade; MUSC musculosidade.

NA - número de animais; NGC - número de grupos de contemporâneos; NAMP - número de animais na matriz de parentesco; DP - desvio padrão; CV - coeficiente de variação, em \%; MIN - valor mínimo; MAX - valor máximo.

W550 - weight at 550 days; WG345 - weight gain from weaning at 550 days; CONF - conformation; PREC - precocity; MUSC - muscling.

$N A$ - number of animals; NCG - number of contemporary groups; NARM - number of animals in relationship matrix; SD - standard deviation; CV - coefficient of variation, in \%; MIN - minimun value; MAX - maximum value. 


\section{Método $\mathfrak{R}$}

Considere que $\mathrm{a}_{\mathrm{i}} \sim \mathrm{N}\left(0, A \sigma_{\mathrm{i}}{ }^{2}\right)$ seja o vetor de efeito aleatório aditivo para a característica i, e A seja a matriz de parentesco. Se $\mathfrak{R}_{\mathrm{i}}$ representa o vetor das soluções estimadas com o conjunto de dados completo e $\Re_{\text {ip }}$, o vetor das soluções estimadas com o conjunto de dados parcial, o fator de regressão $\left(\Re_{c}\right)$ pode ser definido como:

$$
\mathfrak{R}_{\mathrm{c}}=\frac{\hat{\mathrm{a}}_{\mathrm{ip}}^{\prime} \mathrm{A}^{-1} \hat{\mathrm{a}}_{\mathrm{i}}}{\hat{\mathrm{a}}_{\mathrm{ip}}^{\prime} \mathrm{A}^{-1} \hat{\mathrm{a}}_{\mathrm{ip}}}
$$

Segundo Reverter et al. (1994a), o valor esperado da covariância entre estimativas subseqüentes dos valores genéticos aditivos é igual à variância da estimativa prévia, indicando que, em um primeiro momento, o coeficiente de regressão linear iguala a um, indiferentemente da distribuição das observações e predições.

A predição prévia (menos acurada) é obtida pela análise de uma sub-amostra do conjunto total de dados. Se o $\Re_{c}$ for maior que um, então a herdabilidade está sendo subestimada, e se o $\Re_{c}$ for menor que um, a herdabilidade está sendo superestimada. Quando $\Re_{c}$ for igual a um, quer dizer que a precisão foi atingida e então os componentes de variância são utilizados. Neste estudo permitiu-se uma diferença absoluta nos valores de regressão (valor esperado de um) de 0,0002 entre "rounds", ou seja, $1 \pm 0,0002$. Segundo Misztal et al. (1997), com a utilização de uma tolerância de 0,0002 , há um erro numérico correspondente nas estimativas de variâncias de $1 \%$ ou menos da variância total. O principal motivo da tolerância adotada é o ganho na velocidade de processamento sem interferência nos valores dos componentes estimados.

As estimativas de herdabilidade foram obtidas de vinte sub-amostras aleatórias contendo $50 \%$ dos dados, sendo que a amostragem foi feita dentro dos grupos de contemporâneos. A amostragem dentro de grupos de contemporâneos tem como meta reduzir a variância amostrada e também eliminar o viés resultante da seleção sobre os efeitos fixos (Druet et al., 2001). Segundo Reverter et al. (1994b), a amostragem dentro de efeitos fixos aumenta a conectabilidade entre indivíduos, sendo importante na minimização do efeito das variâncias amostradas. O número de subamostras (N) foi suficiente para obtenção de erropadrão baixo $(<0,02)$ da média de herdabilidade
$\left(\overline{\mathrm{h}}_{\mathrm{EP}}^{2}\right)$, que pode ser calculado pela seguinte fórmula $\overline{\mathrm{h}}_{\mathrm{EP}}^{2}=\frac{\mathrm{s}}{\sqrt{\mathrm{N}}}$, em que s é o desvio-padrão das estimativas de herdabilidade.

\section{Intervalos de confiança}

Os intervalos de confiança foram computados para todas as estimativas de herdabilidade realizadas pelo método $\Re$, adotando-se intervalo de confiança a $95 \%$. Os intervalos de confiança têm como objetivo fornecer maior número de informações para a avaliação e descrição das estimativas de herdabilidade oriundas do método $\mathfrak{R}$ (Mallinckrodt et al., 1997). Como as distribuições das herdabilidades não seguiram uma distribuição normal, foi utilizada a transformação Box-Cox para calcular os intervalos de confiança, sendo:

$$
\mathrm{IC}=\overline{\mathrm{X}} \pm \mathrm{t} * \sigma
$$

em que: IC é intervalo de confiança; $\bar{X}$, média da estimativa da herdabilidade transformada; $t$, valor crítico da distribuição t, para um intervalo de confiança de $95 \%$, com graus de liberdade $\mathrm{N}-1$, em que $\mathrm{N}$ é o número de estimativas; $\sigma$, desvio-padrão para as estimativas de herdabilidade transformada para determinado conjunto de dados. Após os cálculos dos intervalos de confiança na escala transformada, os limites foram convertidos de volta para a escala original.

\section{Programas}

Os componentes de variância foram estimados por máxima verossimilhança restrita, utilizando-se o programa MTDFREML - Multiple Trait Derivative Free Restricted Maximun Likelihood (Boldman et al.,1995) em que foi utilizado o algoritmo AI (average information) proposto por Johnson \& Thompson (1995), o qual permite o cálculo dos erros-padrão (Dodenhoff et al., 1998) e pelo Método $\Re$ (Reverter et al., 1994b), utilizando-se o software derivado do BLUP90IOD, do pacote BLUPF90 (Misztal, 1999) com os algoritmos "iteration on data" (IOD) e "preconditioned conjugate gradient" (PCG) (Strandén \& Lidauer, 1999) reescrito por Tsuruta et al. (2001). O critério de convergência adotado foi de $10^{-13}$ para o método REML.

Os resultados obtidos foram comparados pela magnitude das estimativas de herdabilidade com seus respectivos erros-padrão e também pela correlação de Spearman, a qual envolveu os valores genéticos de todos os animais. 
Os componentes de variância obtidos por REML e pelo método $\mathfrak{R}$ foram utilizados para predizer os valores genéticos de todos os animais por BLUP usando as equações de modelos mistos. Os valores ge néticos foram separados por ano de nascimento dos animais para que fossem calculadas as médias anuais. Então, as tendências genéticas foram calculadas pela regressão das médias anuais dos valores genéticos preditos em relação ao ano de nascimento dos animais pelo programa ABTK 2.0 (Golden et al., 1992).

A mudança genética total (MGT) para todas as características foi obtida pela diferença entre as médias dos valores genéticos obtidas nos anos de 1998 e 1988. A mudança genética anual (MGA) foi obtida pela razão entre o MGT e o período de seleção que foi de 10 anos.

\section{Resultados e Discussão}

\section{Estimativas de herdabilidade}

$\mathrm{Na}$ Tabela 2, são mostrados os componentes de variância, os parâmetros genéticos e os intervalos de confiança de $95 \%$ para as características de desempenho ponderal e para os escores visuais estimados pelos métodos REML e $\Re$.

Ao contrário do REML, que trabalha com os valores das variâncias, o método $\Re$ trabalha com razões de variâncias, ou seja, fornece apenas variâncias relativas. Assim, é possível apenas estimar coeficientes de herdabilidade e de correlação genética (não componentes de variância e covariância). Os valores das variâncias residuais obtidas nas aná- lises realizadas por REML foram fixados para o método $\Re$, pois este método não computa variâncias e covariâncias residuais.

Os coeficientes de herdabilidade obtidos para PESSOB foram inferiores ao encontrado por Biffani et al. (1999) e superiores aos valores obtidos por Davis (1993), Eler et al. (1995) e Lôbo et al. (2000), para os dois métodos estudados. Entretanto, os valores obtidos neste estudo foram condizentes ao encontrado por Robinson \& O'Rourke (1992), para o método $\Re$, e ao encontrado por Mercadante et al. (1995), para REML. As estimativas obtidas neste estudo, para ambos os métodos, estão dentro da faixa de valores obtidos por Biffani et al. (1998), Pereira (2001) e Van Melis et al. (2001b). Na Tabela 2, embora os erros-padrão obtidos pelo método $\Re$ sejam iguais a zero, vale chamar a atenção que isto é uma aproximação. Também se verificam que os errospadrão obtidos pelo método $\mathfrak{R}$ foram inferiores aos obtidos por REML.

As estimativas de herdabilidade para GP345 foram superiores às encontradas por Marcondes et al. (2000) e Koury Filho (2001) e dentro da faixa de valores das estimativas obtidas por Lôbo et al. (2000), Pereira (2001) e Silva et al. (2001), tanto para o método REML quanto para o $\Re$.

Para as características de escores visuais, poderia ter sido utilizado um programa específico para dados categóricos (modelo de limiar) que utiliza também a metodologia do método $\mathfrak{R}$, porém como os dados apresentaram uma distribuição próxima da normalidade (simetria) e problemas analíticos de convergên-

Tabela 2 - Estimativas de componentes de variância, parâmetros genéticos e intervalos de confiança para bovinos de corte da raça Nelore

Table 2 - Variance components, genetic parameters and confidence interval estimates for Nellore cattle

\begin{tabular}{|c|c|c|c|c|c|}
\hline & \multirow{2}{*}{\multicolumn{2}{|c|}{$\begin{array}{c}\text { Componentes de variância } \\
\text { Variance components } \\
\text { REML } \\
\text { REML } \\
\end{array}$}} & \multicolumn{2}{|c|}{$\begin{array}{c}\text { Parâmetros genéticos } \\
\text { Genetic parameters }\end{array}$} & \multirow{3}{*}{$\begin{array}{c}\text { IC }(95 \%) \\
C I(95 \%) \\
\text { Método } \Re \\
\text { Method } \Re\end{array}$} \\
\hline & & & \multirow{2}{*}{$\begin{array}{c}\text { REML } \\
\text { REML } \\
\mathrm{h}^{2} \\
\end{array}$} & \multirow{2}{*}{$\begin{array}{c}\text { Método } \Re \\
\text { Method } \Re\end{array}$} & \\
\hline & $\sigma_{\mathrm{a}}^{2}$ & $\sigma_{\mathrm{e}}^{2}$ & & & \\
\hline PESSOB (W550) & 205,60 & 364,33 & $0,36 \pm 0,01$ & $0,39 \pm 0,00$ & 0,36 a 0,42 \\
\hline GP345 (WG345) & 77,50 & 214,77 & $0,27 \pm 0,01$ & $0,29 \pm 0,00$ & 0,27 a 0,33 \\
\hline $\mathrm{CONF}(\mathrm{CONF})$ & 0,15 & 0,53 & $0,22 \pm 0,02$ & $0,21 \pm 0,00$ & 0,17 a 0,24 \\
\hline PREC (PREC) & 0,16 & 0,58 & $0,21 \pm 0,02$ & $0,21 \pm 0,00$ & 0,17 a 0,25 \\
\hline MUSC (MUSC) & 0,16 & 0,56 & $0,22 \pm 0,02$ & $0,22 \pm 0,00$ & 0,19 a 0,25 \\
\hline
\end{tabular}

PESSOB - peso ao sobreano; GP345 - ganho de peso da desmama ao sobreano; CONF - conformação; PREC - precocidade; MUSC musculosidade; REML - máxima verossimilhança restrita; $\sigma_{a}^{2}$ - variância genética aditiva direta; $\sigma_{e}^{2}$ - variância residual; $h^{2}$ - herdabilidade \pm erro-padrão; IC - intervalo de confiança.

W550 - weight at 550 days; WG345 - weight gain from weaning to 550 days; CONF - conformation; PREC - precocity; MUSC - muscling; REML - restricted maximum likelihood; $\sigma_{\mathrm{q}}{ }^{2}$ - direct additive genetic variance; $\sigma_{\mathrm{e}}{ }^{2}$ - residual variance; $h^{2}$ - heritability \pm standard error; $S E$ - standard error; $C l$ - confidence interval. 
cia têm sido encontrados na prática (Evans, 2001, comunicação pessoal), quando o método Réutilizado para estimar componentes de variância para características com muitas classes sob um modelo de limiar, optou-se por utilizar o próprio programa BLUPF90 (Misztal, 1999) para dados contínuos.

Para as características de escores visuais, os coeficientes de herdabilidade obtidos foram inferiores aos encontrados por Eler et al. (1996) e superiores aos encontrados por Jorge Jr. et al. (2001) e Koury Filho (2001). As estimativas de herdabilidade obtidas pelos métodos REML e $\mathfrak{R}$ estão de acordo com as estimativas encontradas por Eler et al. (2001).

Nas análises de escore visual, os resultados de herdabilidade obtidos pelo método $\Re$ não diferiram do método REML, enquanto nas análises para características produtivas, o método $\Re$ apresentou valores de herdabilidade ligeiramente superiores ao REML. Uma provável explicação para este fato é que como o método $\Re$ trabalha com sub-amostras, pode ser que o método sorteie amostras selecionadas, o que resultaria em coeficientes de herdabilidade maiores.

Segundo Cantet \& Birchmeier (1998) e Schenckel $\&$ Schaeffer (2000), as estimativas de herdabilidade pelo método $\mathfrak{R}$ são maiores do que pelo REML, para conjunto de dados selecionados pelo BLUP. Ainda de acordo com os autores, isto ocorre em conseqüência do processo de amostragem que é intrínseco do método, acarretando valores de herdabilidade superestimados. Entretanto, segundo Duangjinda et al. (2001), no trabalho de Cantet \& Birchmeier (1998), obteve-se o viés removido após as relações genéticas entre os animais serem levadas em consideração, tanto que no estudo de Cantet et al. (2000), os autores concluíram que o método $\mathfrak{R}$ forneceu estimativas não viesadas, porém menos consistentes quando comparado com o REML, pelo fato do quadrado médio do erro ou resíduo do método $\mathfrak{R}$ ter sido diversas vezes maior que o do REML. Estes estudos foram realizados com banco de dados simulados, em que a herdabilidade real era conhecida. Para este estudo, não pode ser comprovado se as herdabilidades obtidas pelo método $\mathfrak{R}$ para as características produtivas foram superestimadas pelo fato do não conhecimento do valor exato ou real da herdabilidade, já que o banco de dados é de rebanho comercial. O fato é que alguns valores obtidos pelo método $\Re$ foram ligeiramente maiores que os obtidos pela máxima verossimilhança restrita.

\section{Tendência genética}

Na Tabela 3, são apresentadas as tendências genéticas para as características produtivas e de escore visual, obtidas de análises uni-características pelos métodos REML e $\Re$.

Como mostrado na Tabela 3, os progressos genéticos foram superiores aos valores encontrados por Ferraz Filho et al. (2001) e Van Melis et al. (2001a), e inferiores aos encontrados por Eler et al. (2001).

As mudanças genéticas anuais foram relevantes para todas as características, embora tenham ficado abaixo da cifra de $1 \%$ que significa uma meta a ser atingida na prática (Lôbo et al., 2001).

Em relação aos métodos de estimação REML e $\Re$, nota-se que as diferenças nas estimativas de tendência genética, utilizando os componentes obtidos pelos dois métodos, foram muito pequenas para as características analisadas.

Na Figura 1, são mostradas as tendências genéticas para as características produtivas, obtidas de análises uni-características com os componentes obtidos pelos métodos REML $\mathrm{e} \Re$. As figuras para as características de escore visual não são apresentadas pelo fato de as estimativas de tendência genética terem sido praticamente similares.

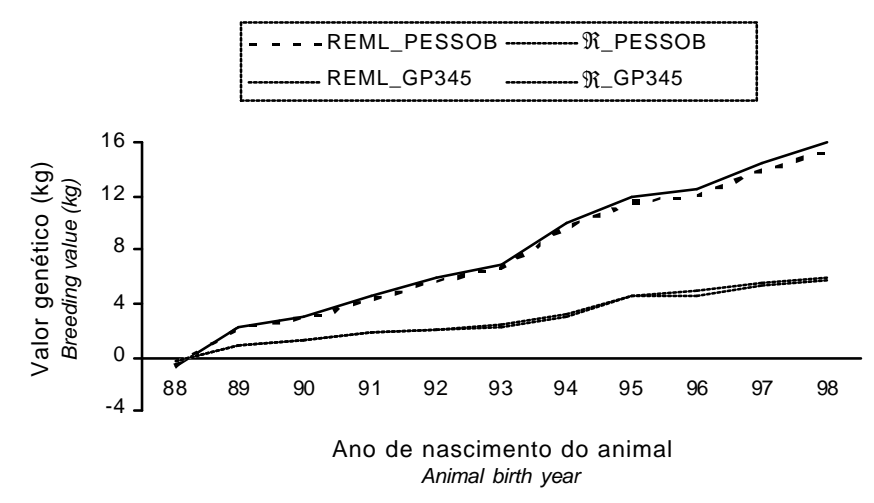

Figura 1 - Tendência genética para peso aos 18 meses e ganho de peso da desmama aos 18 meses, estimadas com os componentes obtidos pelos métodos REML e $\mathfrak{R}$, para bovinos de corte da raça Nelore.

Figure 1 - Genetic trend for weight at 550 days and for weight gain from weaning to 550 days, estimated with variance components obtained by REML and method $\Re$, for Nellore beef cattle. 
Tabela 3 - Tendências genéticas (TG) e mudanças genéticas total (MGT) e anual (MGA) para características produtivas e de escore visual obtidas para bovinos de corte da raça Nelore

Table 3 - Genetic trends (GT) and total and annual genetic changes (TGC and AGC) for productive and visual score traits obtained in Nelore beef cattle

\begin{tabular}{|c|c|c|c|c|c|c|c|c|}
\hline \multirow[t]{2}{*}{$\begin{array}{l}\text { Característica } \\
\text { Trait }\end{array}$} & \multirow[t]{2}{*}{$\begin{array}{l}\text { Método } \\
\text { Method }\end{array}$} & \multirow[t]{2}{*}{$\begin{array}{l}\mathrm{TG} \\
G T\end{array}$} & \multirow[t]{2}{*}{$\begin{array}{l}\mathrm{R}^{2} \\
R^{2}\end{array}$} & \multirow[t]{2}{*}{$\begin{array}{l}1988^{1} \\
1988^{1}\end{array}$} & \multirow[t]{2}{*}{$\begin{array}{l}1998^{2} \\
1998^{2}\end{array}$} & \multirow[t]{2}{*}{$\begin{array}{l}\text { MGT } \\
T G C\end{array}$} & \multicolumn{2}{|c|}{$\begin{array}{c}\text { MGA } \\
A G C\end{array}$} \\
\hline & & & & & & & $\begin{array}{l}\text { Taxa } \\
\text { Rate }\end{array}$ & $\%^{3}$ \\
\hline PESSOB & REML & 1,580 & 0,99 & $-0,66$ & 15,39 & 16,05 & 1,605 & 0,52 \\
\hline$W 550$ & $\mathfrak{K}$ & 1,640 & 0,99 & $-0,70$ & 15,99 & 16,68 & 1,668 & 0,54 \\
\hline GP345 & REML & 0,582 & 0,97 & $-0,27$ & 5,68 & 5,94 & 0,594 & 0,51 \\
\hline WG345 & $\Re$ & 0,611 & 0,97 & $-0,28$ & 5,96 & 6,24 & 0,624 & 0,53 \\
\hline CONF & REML & 0,030 & 0,94 & $-0,00$ & 0,25 & 0,25 & 0,025 & 0,42 \\
\hline CONF & $\mathfrak{R}$ & 0,029 & 0,94 & $-0,00$ & 0,24 & 0,25 & 0,025 & 0,41 \\
\hline PREC & REML & 0,031 & 0,94 & $-0,00$ & 0,25 & 0,26 & 0,026 & 0,43 \\
\hline PREC & $\mathfrak{K}$ & 0,031 & 0,94 & $-0,00$ & 0,25 & 0,26 & 0,026 & 0,42 \\
\hline MUSC & REML & 0,030 & 0,94 & $-0,00$ & 0,24 & 0,24 & 0,025 & 0,42 \\
\hline$M U S C$ & $\mathfrak{R}$ & 0,030 & 0,94 & $-0,00$ & 0,24 & 0,24 & 0,024 & 0,41 \\
\hline
\end{tabular}

PESSOB - peso ao sobreano; GP345 - ganho de peso da desmama ao sobreano; CONF - conformação; PREC - precocidade; MUSC musculosidade; REML - máxima verossimilhança restrita; TG - tendência genética; $R^{2}$ - coeficiente de determinação; ${ }^{1}$ - média dos valores genéticos no ano inicial do cálculo de tendência genética; ${ }^{2}$-média dos valores genéticos no ano final do cálculo de tendência genética; MGT - mudança genética total; MGA - mudança genética anual; ${ }^{3}$ - porcentagem da média para cada característica.

W550 - weight at 550 days; WG345 - weight gain from weaning to 550 days; CONF - conformation; PREC - precocity; MUSC - muscling; REML - restricted maximum likelihood; GT - genetic trend; $P^{2}$ - coefficient of determination; ${ }^{1}$ - mean of genetic values in the initial year to calculate genetic trend; ${ }^{2}$ - mean of genetic values in the final year to calculate genetic trend; TGC - total genetic changed; AGC - annual genetic changed; ${ }^{3}$ - percentage of mean for each trait.

\section{Correlação de Spearman}

O maior interesse das empresas e criadores que necessitam utilizar reprodutores e matrizes é que estes animais sejam avaliados e ordenados corretamente. Assim, é possível maximizar o progresso genético do rebanho e os lucros das propriedades. A utilização da correlação de Spearman tem por objetivo avaliar a consistência da ordenação dos valores genéticos dos animais, utilizando os componentes de variância obtidos pelos dois métodos de estimação.

As correlações de Spearman entre os valores genéticos obtidos em análises utilizando os componentes obtidos pelos métodos REML e R foram iguais a um para todas as características. Isto reflete que ambos os métodos são satisfatórios na obtenção dos componentes de variância a serem utilizados na identificação dos melhores animais em análises uni-características.

\section{Conclusões}

A comparação entre os métodos REML e $\mathfrak{R}$ mostrou que ambos os métodos são consistentes na estimação de herdabilidade, resultando em consistência na predição dos valores genéticos e ordenação dos animais para análises uni-características. Entretanto, o método $\Re$ deve ser utilizado em substituição ao REML somente por razões computacionais, pelo fato de suas propriedades teóricas não serem perfeitamente conhecidas.

\section{Agradecimento}

À Agro-Pecuária CFM Ltda, pelo fornecimento dos dados, e aos pesquisadores Ignacy Misztal e Tom Druet, por autorizarem a utilização do software BLUP90IOD.

\section{Literatura Citada}

BIFFANI, S.; MARTINS FILHO, R.; BOZZI, R. et al. Parâmetros genéticos e fenotípicos para características de crescimento em animais da raça Nelore. In: REUNIÃO ANUAL DA SOCIEDADE BRASILEIRA DE ZOOTECNIA, 35., Botucatu, 1998. Anais... Botucatu: Sociedade Brasileira de Zootecnia, 1998. p.428-430.

BIFFANI, S.; MARTINS FILHO, R.; GIORGETTI, A. et al. Fatores ambientais e genéticos sobre o crescimento ao ano e ao sobreano de bovinos Nelore, criados no nordeste do Brasil. Revista Brasileira de Zootecnia, v.28, n.3, p.468473, 1999.

BOLDMAN, K.G.; KRIESE, L.A.; Van VLECK, L.D. et al. A manual for use for MTDFREML. A set of programs to obtain estimates of variance and covariances [DRAFT]. Lincoln, Departament of Agriculture / Agricultural Research Service, 1995. 120p.

CANTET, R.J.C.; BIRCHMEIER, A.N. The effects of sampling selected data on method $\mathfrak{R}$ estimates of heritability. In: 
WORLD CONGRESS ON GENETICS APPLIED TO LIVESTOCK PRODUCTION, 6., Armidale, 1998. Proceedings... Armidale: WCGALP, 1998. v.25, p.529-532.

CANTET, R.J.C.; BIRCHMEIER, A.N.; SANTOS-CRISTAL, M.G. et al. Comparision of restricted maximum likelihood and method $\Re$ for estimating heritability and predicting breeding value under selection.Journal of Animal Science, v.78, p.2554-2560, 2000.

CULBERTSON, M.S.; MABRY, J.W.; MISZTAL, I. et al. Estimation of dominance variance in purebred Yorkshire swine. Journal of Animal Science, n.76, p.448-451, 1998.

DAVIS, G. P. Genetic parameters for tropical beef-cattle in northern Australia - A Review. Australian Journal of Agricultural Research, v.44, n.2, p.179-198, 1993.

DE ROSE, E.P.; WILTON, J.W.; SCHAEFFER, L.R. Estimation of variance components for traits measured on station-tested beef bulls. Journal of Animal Science, v.66, n.3, p.626634, 1988.

DODENHOFF, J.; Van VLECK, L.D.; KACHMAN, S.D. et al. Parameter estimates for direct, maternal, and grandmaternal genetic effects for birth weight and weaning weight in Hereford cattle. Journal of Animal Science, v.76, p.2521-2527, 1998.

DRUET, T.; MISZTAL, I.; DUANGJINDA, M. et al. Estimation of genetic covariances with Method $\mathfrak{R}$. Journal of Animal Science, v.79, p.605-615, 2001.

DUANGJINDA, M.; MISZTAL, I; BERTRAND, J.K. et al. The empirical bias of estimates by restricted maximum likelihood, Bayesian method, and Method $\Re$ under selection for additive, maternal, and dominance models. Journal of Animal Science, v.79, p.2991-2996, 2001.

ELER, J.P.; FERRAZ, J.B.S.; SILVA, P.R. Estimação de parâmetros genéticos para características produtivas na raça Nelore: II - Fase pós-desmama. In: REUNIÃO LATINOAMERICANA DE PRODUÇÃO ANIMAL, 17 ., Hanava, 2001. Anais... Havana: ALPA, 2001. (CD ROM)

ELER, J.P.; FERRAZ, J.B.S.; SILVA, P.R. Parâmetros genéticos para peso, avaliação visual e perímetro escrotal na raça Nelore, estimados por modelo animal. Arquivo Brasileiro de Medicina Veterinária e Zootecnia, v.48, n.2, p.203213, 1996.

ELER, J.P.; Van VLECK, L.D.; FERRAZ, J.B.S. et al. Estimation of variances due to direct and maternal effects for growth traits of Nellore cattle. Journal of Animal Science, v.73, n.11, p.3253-3258, 1995.

EVANS, J.L. Scores. Mensagem recebida do autor. 22 de Maio de 2001.

FERRAZ FILHO, P.B.; RAMOS, A.A.; SILVA, L.O.C. et al. Tendência genética dos efeitos diretos e materno sobre os pesos à desmama e pós-desmama de bovinos da raça Tabapuã no Brasil. In: REUNIÃO ANUAL DA SOCIEDADE BRASILEIRA DE ZOOTECNIA, 38., Piracicaba, 2001. Anais...Piracicaba: Sociedade Brasileira de Zootecnia, 2001. p.684-685.

GOLDEN, B.L.; SNELLING, W.M.; MALLINCKRODT, C.H. Animal breeder's toolkit: User's guide and reference manual. Colorado: Colorado State University Agricultural Experimental Station Technical Bulletin. LTB92-2. 1992.

JOHNSON, D.L.; THOMPSON, R. Restricted maximum likelihood estimation of variance components for univariate animal models using sparse matrix techniques and average information. Journal of Dairy Science, v.78, p.449-456, 1995.
JORGE JR., J.; EL FARO, L.; ALBUQUERQUE, L.G. Estimativas de herdabilidade para os escores visuais de conformação, precocidade e musculatura, em um rebanho da raça Nelore. In: REUNIÃO ANUAL DA SOCIEDADE BRASILEIRA DE ZOOTECNIA, 38., Piracicaba, 2001. Anais... Piracicaba: Sociedade Brasileira de Zootecnia, 2001. p.618619.

KAISER, C.J.; GOLDEN, B.L. Heritability estimation and breeding value prediction using selected data. Journal of Animal Science, v.72, suppl. 1, abstr. 147, 1994.

KOURY FILHO, W. Análise genética de escores de avaliações visuais e suas respectivas relações com desempenho ponderal na raça Nelore. Pirassununga: Universidade de São Paulo, 2001. 82p. Dissertação (Mestrado) - Universidade de São Paulo, 2001.

LÔBO, R.B.; BEZERRA, L.A.F.; OLIVEIRA, H.N. et al. Avaliação genética de animais jovens, touros e matrizes: Sumário do PMGRN - 2001. 50p.

LÔBO, R.N.B.; MADALENA, F.E.; VIEIRA, A.R. Average estimates of genetic parameters for beef and dairy cattle in tropical regions. Animal Breeding Abstract, v.68, n.6, p.433-462, 2000.

MALLINCKRODT, C.H.; GOLDEN, B.L.; REVERTER, A. Approximate confidence intervals for heritability fom Method $\mathfrak{R}$ estimates. Journal of Animal Science, v.75, p.2041-2046, 1997.

MARCONDES, C.R.; BERGMANN, J.A.G.; ELER, J.P. et al. Análises de alguns critérios de seleção para características de crescimento na raça Nelore. Arquivo Brasileiro de Medicina Veterinária e Zootecnia, v.52, n.1, p.83-89, 2000.

MERCADANTE, M.E.Z.; LÔBO, R.B.; REYES, A. de los. Parámetros geneticos para características de crecimiento em cebuínos de carne: uma revisión. Archivos Latinoamericanos Producción Animal, v.3, p.45-89, 1995.

MISZTAL, I. Estimation of variance components with largescale dominance models. Journal of Dairy Science, v. 80, p. 965-974, 1997.

MISZTAL, I. Complex models, more data: Simpler programming. In: INTERNATIONAL WORKSHOP COMPUTATION CATTLE BREED, 1999, Tuusula, Finland. Proceedings... Tuusula: Interbull Bull, 1999. v.20, p.33-42.

MISZTAL, I.; LAWLOR, T.J.; FERNANDO, R.L. Dominance models with method $\mathrm{R}$ for stature of Holstein. Journal of Dairy Science, v.80, p.975-978, 1997.

NELSEN, T.C.; SHORT, R.E.; URICK, J.J. et al. Heritabilities and genetic corelations of growth and reproductive measurements in Hereford bulls. Journal of Animal Science, v.63, p.409-417, 1986.

PEREIRA, E. Análise genética de algumas características reprodutivas e de suas relações com desempenho ponderal na raça Nelore. Pirassununga: Universidade de São Paulo, 2001. 56p. Dissertação (Mestrado em Qualidade e Produtividade Animal) - Universidade de São Paulo, 2001.

REVERTER, A.; GOLDEN, B.L.; BOURDON, R.M. et al. Technical note: detection of bias in genetic predictions. Journal of Animal Science, v.72, p.34-37, 1994a.

REVERTER, A.; GOLDEN, B.L.; BOURDON, R.M. et al. Method $\mathfrak{R}$ variance components procedure: application on the simple breeding value model. Journal of Animal Science, v.72, p.2247-53, 1994b.

ROBINSON, D.L.; OROURKE, P.K. Genetic parameters for liveweights of beef cattle in the tropics. Australian Journal of Agricultural Research, v.43, n.6, p.1297-1305, 1992. 
SCHAEFFER, L.R. Variance component estimation: course notes. Armidale: University of New England, Animal Genetics and Breeding Unit, 1998. 155p.

SCHAEFFER, L.R.; WILTON, J.W. Estimation of variances and covariances for use in a multiple trait beef sire evaluation model. Canadian Journal of Animal Science, v.61, p.531538, 1981

SCHENKEL, F.S.; SCHAEFFER, L.R. Effects of nonrandom parental selection on estimation of variance components. Journal Animal Breeding Genetics, v.177, p.225-239, 2000.

SILVA, J.A. II V.; Van MELIS, M.H.; ELER, J.P. et al. Análise multivariada de características produtivas em bovinos da raça Nelore - Parâmetros genéticos. In: REUNIÃO LATINOAMERICANA DE PRODUÇÃO ANIMAL, 17., Havana, 2001. Anais... Havana: ALPA, 2001. (CD ROM)

STRANDÉN, I.; LIDAUER, M. Solving large mixed linear models using preconditioned conjugate gradient iteration. Journal of Dairy Science, v.82, p.2779-2787, 1999.

TESS, M.W.; JESKE, K.E.; DILLARD, E.U. et al. Sire $\mathrm{x}$ environment interactions for growth traits of Hereford cattle. Journal of Animal Science, v. 59, p. 1467-1476, 1984.

TSURUTA, S.; MISZTAL, I.; STRANDÉN, I. Use of precontioned conjugate gradient algorithm as a generic solver for mixed-model equations in animal breeding applications. Journal of Animal Science, v.79, p.1166-1172, 2001.
Van MELIS, M.H.; ELER, J.P.; FERRAZ, J.B.S. Tendências genéticas para características produtivas e de avaliação visual em bovinos da raça Nelore. In: REUNIÃO ANUAL DA SOCIEDADE BRASILEIRA DE ZOOTECNIA, 38., Piracicaba, 2001.Anais...Piracicaba: Sociedade Brasileira de Zootecnia, 2001a. p.519-520.

Van MELIS, M.H., SILVA, J.A. II de V.; ELER, J.P. et al. Estimação de herdabilidade para características pós-desmama pelos métodos REML e $\mathfrak{R}$. In: REUNIÃO LATINOAMERICANA DE PRODUÇÃO ANIMAL, 17., Hanava, 2001. Anais... Havana: ALPA, 2001b. (CD ROM)

WRIGTH, H.B.; POLLAK, E.J.; QUAAS, R.L. Estimation of variance and covariance components to determine heritabilities and repeatability of weaning weight in American Simmental cattle. Journal of Animal Science, v.65, p.975-981, 1987.

Recebido em: 01/04/02 Aceito em: 08/03/03 